\title{
Correction: 0-GIcNAcylation promotes colorectal cancer metastasis via the miR-101-0-GIcNAc/EZH2 regulatory feedback circuit
}

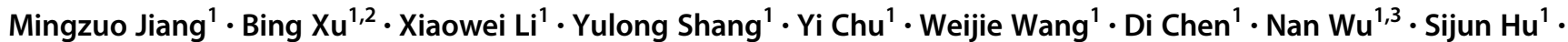

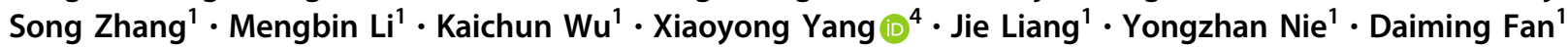

Published online: 3 June 2019

(c) The Author(s), under exclusive licence to Springer Nature Limited 2019

Correction to: Oncogene (2019) 38:301-316 https://doi.org/10.1038/s41388-018-0435-5

In the original version of this article the authors noted that they accidentally misplaced data belonging to Figure $2 \mathrm{~h}$ (migration of TMG treatment group) into Fig. 5h (migration of miR-101 inhibitor transfection group) when re-editing the figure to meet the format and the image resolution of the journal. The amended version of Fig. $5 \mathrm{~h}$ is provided below. This change does not affect the results or conclusions reported in the manuscript.
These authors contributed equally: Mingzuo Jiang, Bing Xu, Xiaowei Li, Yulong Shang

Yongzhan Nie

yongznie@fmmu.edu.cn

$\triangle$ Daiming Fan

daimingfan@fmmu.edu.cn

1 State Key Laboratory of Cancer Biology, National Clinical Research Center for Digestive Diseases and Xijing Hospital of Digestive Diseases, Fourth Military Medical University,

Xi'an, China
2 Department of Gastroenterology, Second Affiliated Hospital of Xi' an Jiaotong University, Xi' an 710004 Shaanxi Province, China

3 Lab of Tissue Engineering, Faculty of Life Science, Northwest University, Xi'an, China

4 Department of Molecular Cellular and Developmental Biology, Yale University, New Haven, USA 
Fig. $1 \mathbf{h}$ Transwell assay of SW480 or SW620 cells with the indicated treatment. *represents Student's t test $P<0.05$ and $* * P<0.01$
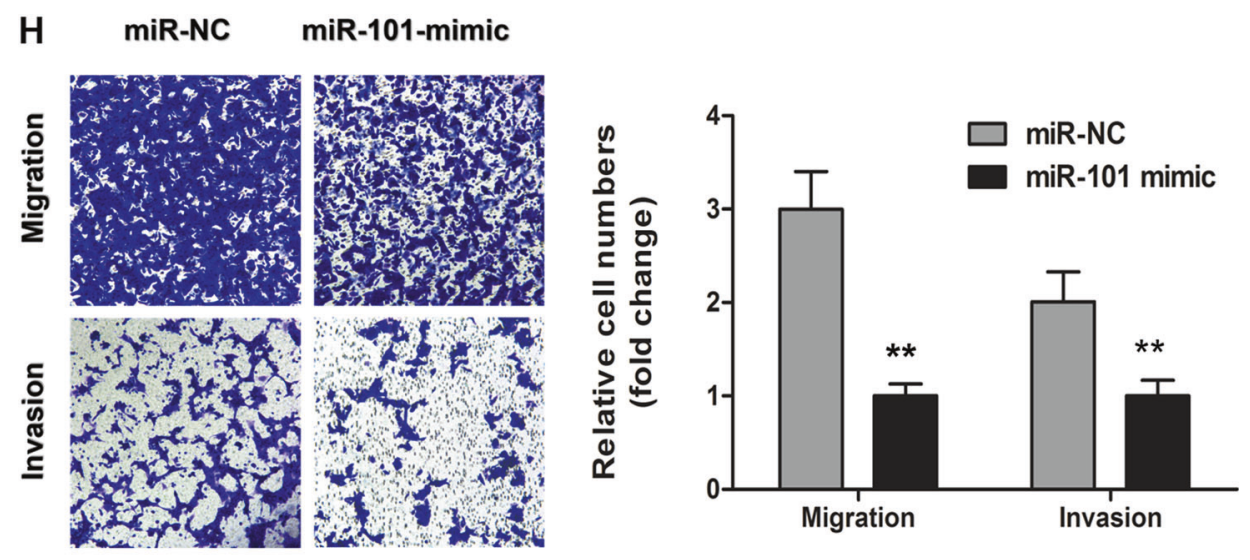

miR-NC miR-101-inhibitor
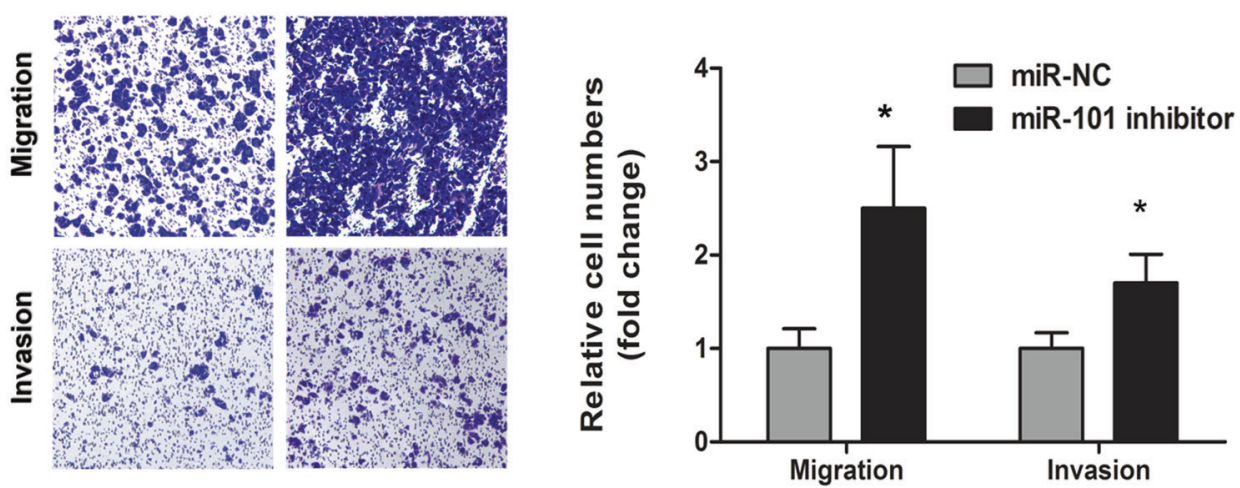